\title{
Surface Vibration Reconstruction using Inverse Numerical Acoustics
}

\author{
F. Martinus, D. W. Herrin \& A. F. Seybert \\ Department of Mechanical Engineering, University of Kentucky \\ 151 RGAN Building, Lexington, KY 40506-0503, USA
}

\begin{abstract}
This paper explores the use of inverse numerical acoustics to reconstruct the surface vibration of a noise source. Inverse numerical acoustics is mainly used for source identification. This approach uses the measured sound pressure at a set of field points and the Helmholtz integral equation to reconstruct the normal surface velocity. The number of sound pressure measurements is considerably less than the number of surface vibration nodes. An overview of inverse numerical acoustics is presented and compared with other holography techniques such as nearfield acoustical holography and the Helmholtz equation least squares method. In order to obtain an acceptable reproduction of the surface vibration, several critical factors such as the field point selection and the effect of experimental errors have to be handled properly. Other practical considerations such as the use of few measured velocities and regularization techniques will also be presented. Examples will include a diesel engine, a transmission housing and an engine cover.
\end{abstract}

Keywords: inverse numerical acoustics; surface vibration reconstruction; INA; acoustic holography; inverse BEM; acoustic transfer vector.

\section{$1 \quad$ Introduction}

The most widely used numerical method to predict sound radiated by vibrating structures has been the boundary element method (BEM) [1]. A BEM analysis requires the surface vibration as an input. Traditional noise prediction approaches use either a structural finite element analysis or measurements to obtain this surface vibration. However, it takes considerable effort to build a finite element model or to run experiments on large and complex models. To alleviate the difficulties related to the traditional approach, several reconstruction approaches such as nearfield acoustical holography (NAH), the HELS method, and inverse numerical acoustics (INA) have been developed to reconstruct the surface vibration. The advantages and disadvantages of different methods were discussed in a paper by the authors [2].

Inverse numerical acoustics techniques using both the direct and indirect boundary element methods have been investigated previously [3-9]. In INA, the goal is to solve for the surface normal velocities using measured field point 
sound pressures. For practical reasons, it is beneficial to utilize a minimal number of field points for collecting information about the acoustic field. Therefore, the number of field points is normally less than the number of nodes where velocities are to be computed. This results in an ill-posed problem, in which the solution is not unique on a practical level and does not depend continuously on the measured data. In practice this means that INA is sensitive to measurement errors unless suitable stabilizing constraints are imposed. In experimental data such errors and noise are of course inevitable and thus some information about the sound source is lost due to these errors. Therefore, the challenge is to solve an INA problem in the face of lost information and experimental errors.

\section{$2 \quad$ Mathematical Formulation}

\subsection{Acoustic Transfer Matrix}

The direct boundary element method is based on the Helmholtz integral equation which is expressed as [1]:

$$
\begin{aligned}
& p_{F}=-\int_{S}\left(i \rho \omega G v_{n}+p_{s} \frac{\partial G}{\partial n}\right) d S \\
& G(r)=\frac{e^{-i k r}}{4 \pi r}
\end{aligned}
$$

where $p_{F}$ is the complex sound pressure in the field of a source, $G$ is the free space Green's function, $n$ is the unit normal on the surface of the radiating body $S$ directed away from the acoustic domain. $v_{n}$ and $p_{s}$ are the surface normal velocity and sound pressure on $S$, respectively. Eq. (1) can be evaluated on the surface and a system of equation relating the surface sound pressure and the surface normal velocity can be obtained:

$$
\left[H_{s}\right]\left\{p_{s}\right\}=\left[G_{s}\right]\left\{v_{n}\right\} \rightarrow\left\{p_{s}\right\}=\left[H_{s}\right]^{-1}\left[G_{s}\right]\left\{v_{n}\right\}
$$

where $\left[H_{s}\right]$ and $\left[G_{s}\right]$ are $\mathrm{NxN}$ matrices, where $\mathrm{N}$ is the number of nodes on the surface. By evaluating Eq. (1) in the field, and combining with Eq. (2), the relation between sound pressure in the field and the surface normal velocities can be written as:

$$
\begin{aligned}
& \left\{p_{f}\right\}=\left\lfloor H_{f}\right\rfloor\left\{p_{s}\right\}+\left\lfloor G_{f}\right\rfloor\left\{v_{n}\right\} \\
& \left\{p_{f}\right\}=\left(\left[H_{f}\right]\left[H_{s}\right]^{-1}\left[G_{s}\right]+\left[G_{f}\right]\right)\left\{v_{n}\right\}=[A T M]\left\{v_{n}\right\}
\end{aligned}
$$

where $[A T M]$ is the acoustic transfer matrix. For a single field point, Eq. (3) is: 


$$
p_{F, i}=\left[\underline{A T V}_{i}^{T}\right]\left\{v_{n}\right\}
$$

where $\left[\underline{A T V}_{i}^{T}\right]$ is the $\mathrm{i}^{\text {th }}$ row of the $[A T M]$. This vector is often referred to as the acoustic transfer vector [9], acoustic contribution vector, or acoustic sensitivity vector. It is an ensemble of acoustic transfer functions relating the normal velocity of each node to the sound pressure at a single microphone location. The concept of the ATV is illustrated in Figure 1.

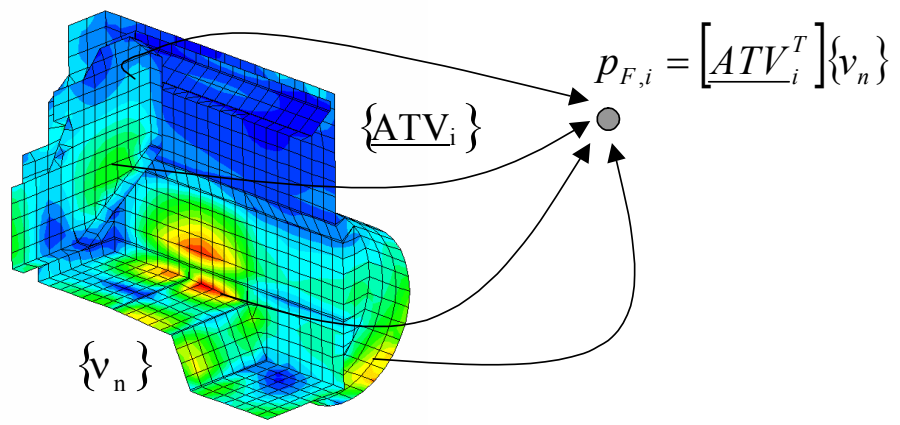

Figure 1 Acoustic transfer vector concept.

Although in the preceding development the $[A T M]$ was derived using the direct boundary element method (DBEM), it can also be derived using other numerical methods such as the indirect boundary element method (IBEM), acoustic FEM, the Rayleigh integral, the high frequency boundary element method (HFBEM), and also measurements, as long as a relationship between the output (field point pressures) and the input (structural vibration) can be obtained.

\subsection{Inverse Numerical Acoustics (INA)}

In INA, the goal is to solve Eq. (3) for the normal velocities $\left\{v_{n}\right\}$ using measured field point sound pressures $\left\{p_{F}\right\}$. In practice, the number of field points NF is normally less than the number of nodes $\mathrm{N}$ where velocities are reconstructed.

The velocity boundary conditions $\left\{v_{n}\right\}$ are computed by employing a singular value decomposition (SVD) algorithm [10] for solving Eq. (3). The SVD algorithm is needed because $[A T M]$ is not a square matrix, and, therefore, $[A T M]^{-1}$ does not exist. Since NF $<\mathrm{N}$, the SVD is normally defined by considering $A=[A T M]^{\mathrm{T}}$, where the columns of $A$ correspond to the different field point locations. Consequently, the ATV's for each field point are now the columns of $A$. Using SVD, $A$ can be expressed as: 


$$
\begin{array}{r}
{\left[\operatorname{ATM}_{N F x N}\right]^{T}=A_{N x N F}=[U]_{N x N F}[\Sigma]_{N F x N F}[V]_{N F x N F}^{T}} \\
{[\Sigma]=\left[\begin{array}{lll}
\sigma_{1} & & \\
& \ddots & \\
& & \sigma_{N F}
\end{array}\right], N>N F}
\end{array}
$$

where $[U]$ and $[V]$ are orthogonal matrices satisfying $[U]^{\mathrm{T}}[U]=[V]^{\mathrm{T}}[V]=[V][V]^{\mathrm{T}}=[\mathrm{I}]$. The $\sigma_{\mathrm{i}}$ 's are the singular values of $A$. Once the SVD of $A$ has been computed, the surface normal velocity can be reconstructed by:

$$
\begin{gathered}
{[A T M]\left\{v_{n}\right\}=\left\{p_{F}\right\} \Rightarrow[V][\Sigma][U]^{T}\left\{v_{n}\right\}=\left\{p_{F}\right\}} \\
\Rightarrow\left\{v_{n}\right\}=[A T M]^{+}\left\{p_{F}\right\}
\end{gathered}
$$

where $[A T M]^{+}=[U][\Sigma]^{+}[V]^{T}$ is called the Moore-Penrose pseudo-inverse of $[A T M][10]$.

\subsection{Other Holographic Reconstruction Techniques}

Nearfield Acoustic Holography (NAH). In nearfield acoustic holography (NAH), the measurement of the sound pressure field on a two-dimensional surface is used to determine the three-dimensional sound pressure field, the particle velocity field, the sound intensity field, the surface velocity, etc. [11,12]. Measurements are usually made on a planar surface (the hologram plane), and these data are used to uniquely reconstruct the three-dimensional field. Due to the exponential decay of the evanescent wave, the NAH measurement is normally done in the nearfield region [11,12].

The NAH algorithm works under the assumption that there is a Green's function that satisfies a homogeneous condition on the source surface. Therefore, NAH only works well for sources with simple geometry (planar or cylindrical) $[11,12,13]$. In order to take into account the evanescent field, the hologram surface on which measurements are taken must be very close to the source surface, within one-half wavelength [14].

The HELS Method. In the HELS method, reconstruction of the acoustic field is done by directly solving the Helmholtz differential equation [15-17]. The acoustic pressure is expanded in a set of independent functions generated by the Gram-Schmidt orthonormalization, and the coefficients associated with these independent functions are determined by requiring the assumed form of solution to satisfy a sound pressure boundary condition at the measurement points. The errors incurred in this process are minimized by the least squares method. Once these coefficients are determined, the acoustic pressure anywhere, including on 
the source surface, may be calculated. Since the number of expansion terms determines the number of measurements, which is usually much smaller than the number of discretized nodes on the surface, reconstruction of the acoustic pressure field can be done efficiently; however, surface vibration can not be obtained directly. The HELS method is particularly suitable for spheroidal geometry.

\section{$3 \quad$ Numerical Considerations}

\subsection{Uniqueness of the Solution}

INA solves an underdetermined problem. Thus, the solution $\left\{v_{n}\right\}$ is not unique because any linear combination of vectors from the null space of $A$ can be added to the solution. This can be explained by examining the SVD of $A$ expressed in Eq. (5). For the case where $\mathrm{N}=\mathrm{NF}$ and $A$ has a full $\operatorname{rank}$, i.e. $\operatorname{rank}(A)=\mathrm{N}$ and $\sigma_{1} \geq \sigma_{2} \geq \ldots \geq \sigma_{N}>0$, the problem reduces to solving a system of linear equations. In this case, the solution is unique. However, since in practice $A$ is a rank deficient matrix, i.e., $\operatorname{rank}(A)=\mathrm{r}<\mathrm{N}$, only $\mathrm{r}$ singular values of $A$ are not equal to zero.

The SVD of $A$ can then be expressed as:

$$
\begin{aligned}
& A=\left[\begin{array}{lllllll}
\underline{u}_{1} & \underline{u}_{2} & \cdots & \underline{u}_{r} & \underline{u}_{r+1} & \cdots & \underline{u}_{N F}
\end{array}\right] *\left[\begin{array}{cccccc}
\sigma_{1} & & & & \\
& \ddots & & & \\
& & \sigma_{r} & & \\
& & & 0 & \\
& & & \ddots & \\
& & & & \\
& & & & 0
\end{array}\right] *\left[\begin{array}{c}
V_{1}^{T} \\
\underline{V}_{2}^{T} \\
\vdots \\
\underline{V}_{r}^{T} \\
\vdots \\
\underline{V}_{N F}^{T}
\end{array}\right] \\
& A\left[\begin{array}{llllll}
\underline{V}_{1} & \cdots & \underline{V}_{r} & \underline{V}_{r+1} & \cdots & \underline{V}_{N F}
\end{array}\right]=\left[\begin{array}{llllllll}
\sigma_{1} \underline{u}_{1} & \sigma_{2} \underline{u}_{2} & \cdots & \sigma_{r} \underline{u}_{r} & 0 & \cdots & 0
\end{array}\right]
\end{aligned}
$$

It can be seen from Eq. (7) that vectors $\underline{V}_{j}$ with $\mathrm{j}>\mathrm{r}$ are elements of the null space of $A$ that satisfy $A \underline{V}_{j}=0$, and, therefore, if $\{\underline{x}\}$ is a solution to $A \underline{x}=\underline{b}$ then $\left\{\underline{x}+t \underline{V}_{j}\right\}$ (t is a constant) is also a solution. Hence the solution to Eq. (3) for a rank deficient matrix $A$ is not unique. The physical explanation is that there might be several different sets of $\left\{v_{n}\right\}$ that produce the same values at $\left\{p_{F}\right\}$ since in practice $\mathrm{NF}<\mathrm{N}$.

Since INA solves an underdetermined problem, Eq. (3) can be highly sensitive to errors. The primary difficulty is due to the cluster of small singular values in [ATM]. Hence, some further information about the desired solution is 
necessary to stabilize the problem. There are many different procedures that can be implemented, usually referred to as regularization methods. One of the most common and well-established regularization procedures is Tikhonov regularization [18]. The method is based on the observation that illconditioning usually results in widely dispersed values in the solution vector. Therefore, it is required that besides having a possible close approximation of the theoretical solution, the 2-norm of the solution itself should be small. The solution sought is $\left\{\mathrm{v}_{\mathrm{n}}\right\}=\left\{\mathrm{v}_{\mathrm{n}}\right\}_{\text {Tikh }}$ that minimizes the weighted combination of the residual norm $\left\|[A T M]\left\{v_{n}\right\}-\left\{p_{F}\right\}\right\|$ and the side constraint $\left\|[L]\left\{v_{n}-v_{0}\right\}\right\|$ :

$$
\left\|[A T M]\left\{v_{n}\right\}-\left\{p_{F}\right\}\right\|_{2}^{2}+\lambda^{2}\left\|[L]\left\{v_{n}-v_{0}\right\}\right\|_{2}^{2}
$$

where $[\mathrm{L}]$ is the identity matrix or a discrete approximation of a derivative operator, $v_{0}$ is an initial estimate of the solution, and $\lambda$ is a regularization parameter. The higher the regularization parameter, the larger the amount of regularization (smaller solution norm at the cost of a larger residual norm) and vice versa. An optimum selection for $\lambda$ can be obtained by plotting the residual norm versus the solution norm (side constraint) in a log-log scale. This curve is normally known as the L-curve [19].

\subsection{Accuracy of the Reconstruction}

If $\left\{v_{n}\right\}$ is the actual solution that minimizes $\left\|[A T M]\left\{v_{n}\right\}-\left\{p_{F}\right\}\right\|_{2}$, the computed solution $\left\{\widetilde{v}_{n}\right\}$ contains errors as a result of the INA process. The reconstruction error can be in general written as [10]:

$$
\frac{\left\|\tilde{v}_{n}-v_{n}\right\|_{2}}{\|v\|_{2}}=C \cdot \kappa_{2}(A)\left[\frac{\|\delta A\|_{2}}{\|A\|_{2}}+\frac{\left\|\delta p_{F}\right\|_{2}}{\left\|p_{F}\right\|_{2}}\right]
$$

where $C$ is a constant and $\kappa_{2}(A)$ is the condition number of matrix $A$. Eq. (9) suggests that any errors in the process of building matrix $A$ and measurements of $\left\{p_{F}\right\}$ will be amplified by $\kappa_{2}(A)$.

Based on Eq. (9), several factors that are critical in producing error can be categorized. These factors will be examined in the following discussion.

Error in the [ATM]. This comes from the forward acoustic analysis in determining the relation or transfer function between the normal velocity at each node and the field point pressure at a field point. If this step is done using the DBEM, then the error could come from solving Eq. (2) and (3). Therefore, it is important to note that in computing the $[A T M]$, the boundary element 
model must accurately simulate the radiating body under consideration, i.e., the model must be accurate.

Error in field point sound pressure measurements $\left\{\boldsymbol{p}_{\mathrm{F}}\right\}$. Since in most cases $\left\{p_{F}\right\}$ are complex and are obtained from measurements, the error can be divided into magnitude and phase errors. Therefore, the calibration of microphones and sensors, the selection of a stable reference, and the accuracy of microphone placing all play a critical role in minimizing the error in $\left\{p_{F}\right\}$.

The condition number of $A$. Since $A$ is not square, $\kappa_{2}(A)$ with respect to the 2norm is defined as:

$$
\kappa_{2}(A)=\|A\|_{2}\left\|A^{+}\right\|_{2}=\frac{\sigma_{\text {max }}(A)}{\sigma_{\text {min }}(A)}
$$

From Eq. (10) it can be observed that $A$ is very ill-conditioned when there are clusters of small singular values (compared to the largest singular value). A regularization technique can be implemented to discard these small singular values by applying an SVD tolerance $\alpha$. This process is called truncated singular value decomposition. The singular values are dropped and set to zero as soon as $\sigma_{\mathrm{i}}<\alpha \sigma_{1}$. This process creates a new matrix:

$$
A_{k}=U \Sigma_{k} V^{T}, \Sigma_{k}=\left[\begin{array}{ccccccc}
\sigma_{1} & & & & & \\
& \ddots & & & & \\
& & \sigma_{k} & & & \\
& & & 0 & & \\
& & & & \ddots & \\
& & & & & 0
\end{array}\right]
$$

where it can be proved that $A_{k}$ is the matrix closest to $A$ among all matrices having rank $k[10]$. it can also be shown that:

$$
\left\|A-A_{k}\right\|_{2}=\sigma_{k+1}
$$

Since each singular value $\sigma_{i}$ corresponds to a column $\underline{u}_{i}$ of $[U]$, setting a singular value to zero is the same as eliminating the contribution from a field point $p_{F, i}$. Thus, by applying an SVD tolerance, field points that contribute to the small singular values are deleted, the resulting condition number is lower, and $A$ becomes well conditioned. However, it has to be noted that since rank of $A$ equals the column space of $[U]$, the resulting matrix $A_{k}$ has a lower rank each time a singular value is discarded. As an example, if originally a set of NF field points (corresponding to NF non-zero singular values) are used, and if after applying the truncated SVD process $m$ singular values are discarded, the 
resulting matrix $A_{k}$ is well conditioned but has a rank $k=(N F-m)$, and, hence, is more rank deficient. Therefore, the choice of $\alpha$ is a trade-off between regularization and loss of information and the critical issue of this approach is to correctly select $\alpha$ such that the errors are sufficiently reduced without causing unacceptable loss of information.

The information content in $\boldsymbol{A}$. As has been mentioned above, the truncated SVD process produces a well conditioned $A_{k}$ which is the closest approximation to $A$. However, $A$ may not have sufficient information content to model the sound field to begin with. High information content means that $A$ has a large number of linearly independent columns. This is highly dependent on the selection of field points and will be discussed on the next section of this paper. If $\operatorname{rank}(A)<<\mathrm{N}, A$ is highly rank deficient and may not produce an accurate reconstruction particularly for complex source.

\section{$4 \quad$ Practical Considerations}

\subsection{Field Point Selection}

The selection of microphone locations has been one of the primary issues in INA implementation. The main issues include selecting an appropriate shape, distribution, and number of field points to accurately reconstruct the surface vibration. There have been few formal guidelines dealing with defining the parameters mentioned above. However, as a guideline, field points are selected to collect information about the sound field. Therefore, for best results, field points should be selected so that the information content is unique and complete.

Uniqueness means that each field point must provide unique information about the sound field. Problems arise when two or more field points capture essentially the same or similar information. An example is illustrated in Figure 2 for two points close together but are far away from the source.

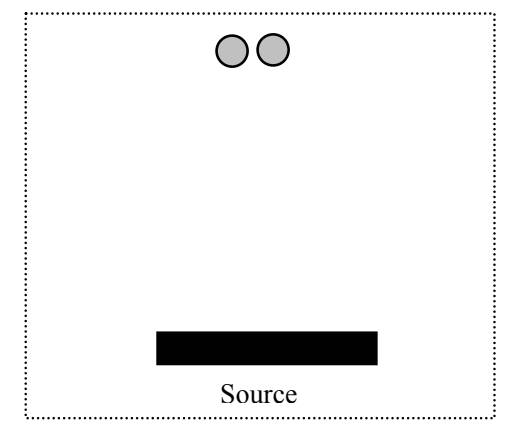

Figure 2 Field points with non-uniqueness problem. 
Numerically, if matrix $A$ only has $r$ unique field points correspond to it, there will only be $r$ linearly independent columns in $A$. Then, $\sigma_{r+1} \ldots \sigma_{N F}$ will be a cluster of small numbers and will be set to zero after applying the SVD tolerance. Having a large number of non-unique field points will not help the reconstruction since only the unique field points will be kept and the rest will be discarded. It can be shown that field points located either too far from the source or do not follow the surface curvature produce this non-uniqueness problem.

Completeness means that the field points capture 'enough' information. An example where a completeness problem might arise is shown in Figure 3. The solution will not exist since the behavior of the source is not completely known.

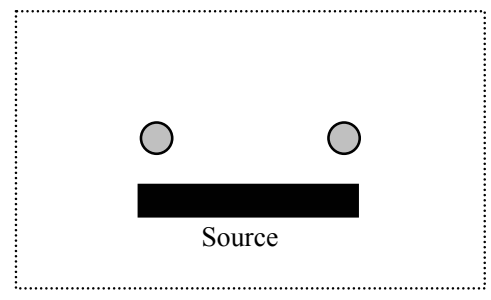

Figure 3 Field points with completeness problem.

A set of field points that does not capture complete information about the sound source will result in a highly rank-deficient matrix $A$; the solution will not be unique and may in fact converge to a wrong solution.

\subsection{Effect of Experimental Errors}

Eq. (9) indicates that experimental errors will be particularly critical when $\kappa_{2}(A)$ is large or when $A$ is ill-conditioned. In fact, changing $\left\{p_{F}\right\}$ to $\left\{p_{F}+\delta p_{F}\right\}$ can change $\left\{v_{n}\right\}$ to $\left\{v_{n}+\delta v_{n}\right\}$ where:

$$
\left\|\delta v_{n}\right\|_{2} \approx \frac{\left\|\delta p_{F}\right\|_{2}}{\sigma_{\min }}
$$

with $\sigma_{\min }$ is the smallest singular value of $A$ assuming $\sigma_{\min }>0$ [10].

\section{$5 \quad$ Suggestions for Improving Ina Results}

\subsection{Partial Reconstruction}

In cases where the sound source is complex, it is often advantageous to reconstruct the velocities not on all the surfaces but only on the particular 
surface of interest. In partial reconstruction, the sound pressure at the field points are assumed to be related only to the vibration on the particular surface of interest, the other surfaces are assumed to be noncontributing to the field point pressure. This assumption requires that field points are placed near the particular surface of interest. No field points are needed in the near-field region of the other surfaces. Therefore, the contribution from the vibration at the nodes located on those surfaces can be removed from the $[A T M]$. The saving in computing effort comes from two main factors: (1) the size of the resulting $[A T M]$ is much smaller, (2) The rank of $A$ necessary to obtain a reasonable reconstruction is lower, meaning that the number of field points needed for the reconstruction is also smaller, hence, simplify the algorithm for field point selection process.

\subsection{Using A Few Measured Surface Vibration Data}

If the velocities on some of the surface nodes are known then Eq. (3) can be rewritten as:

$$
\begin{aligned}
& \left\{p_{F}\right\}=\left[A T M_{m} A T M_{u}\right]\left\{\begin{array}{c}
v_{n}^{m} \\
v_{n}^{u}
\end{array}\right\} \\
& \left\{p_{F}\right\}^{*}=\left\{p_{F}\right\}-\left[A T M_{m}\right]\left\{v_{n}^{m}\right\}=\left[A T M_{m}\right]\left\{v_{n}^{u}\right\}
\end{aligned}
$$

Where the index $m$ corresponds to the measured data and $u$ corresponds to what is unknown. We can immediately observe two advantages using this approach: (1) the surface velocities at fewer points will need to be reconstructed, and (2) the measured velocities can be used to regularize the solution (Eq. (8)). In practice, only a few measured surface vibration data is needed to regularize the solution. Therefore, even in cases where one can measure the entire surface vibration directly, this approach is still advantageous since it requires a significantly less measurement and analysis time. As an illustration, measuring 1814 vibration nodes on the Cummins diesel engine shown in Figure 4 took about 2 weeks to complete the entire measurements. On the other hand, the reconstruction of surface vibration using the INA and 10 measured vibration data was completed in one day.

\section{Test Cases}

\subsection{No Experimental Error}

Models. Two different models are used in this paper. The first model is that of a B-series Cummins diesel engine. The engine and the associated mesh are shown in Figure 4. The engine running condition was $2000 \mathrm{rpm}$ at no load. The element length for each element was approximately $50 \mathrm{~mm}$, resulting in 
1814 nodes. The BEM mesh was painted on the engine, and then vibration measurements were made at each of the points on the engine surface. After the vibration was measured, the sound power of the engine was determined using the sound intensity method. Therefore, the actual surface velocities and radiated sound power are known.

For INA calculations, the field point sound pressures were not obtained from measurements but from a forward BEM analysis using the measured surface vibration as the boundary condition. Consequently the field point sound pressure data have no contamination due to experimental errors.
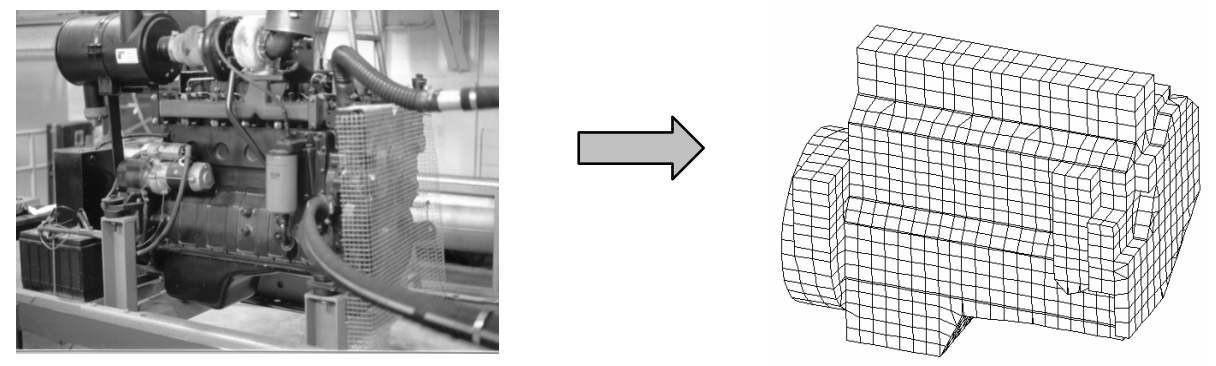

Figure 4 Cummins diesel engine and the corresponding BEM mesh.

The second model is a transmission housing. The transmission housing differed from the first case since it is long and more cylindrical (less box like) and the response is more tonal in character (very few modes). In this case the surface velocities were obtained from an FEM analysis. Free-free boundary conditions were assumed and a forced response analysis was used to predict the vibration. The radiated sound power was obtained from a forward BEM analysis. The BEM model of the transmission housing is shown in Figure 5.

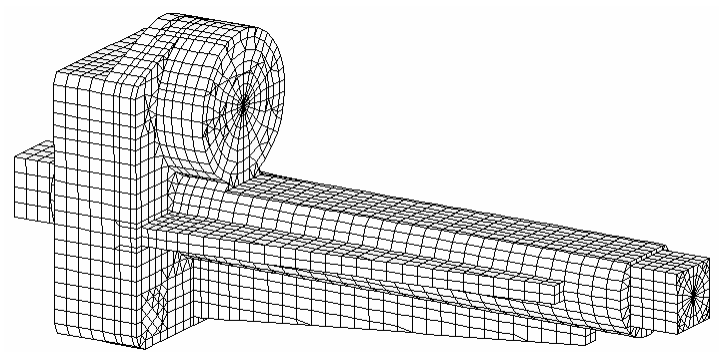

Figure 5 BEM mesh of the transmission housing.

Effect of Field Point Selection. A 152 field point sphere was used for both the engine (shown in Figure 6) and the transmission housing. In addition, six field point planes were defined for the engine (128 points) and the transmission 
housing (66 points), shown in Figures 7 and 8. The reconstructed surface vibration for both cases is shown in Figures 9 and 10 . The reconstructed surface vibrations were then used in a forward BE analysis to obtain the radiated sound power. This radiated sound power was compared to the actual sound power obtained from measurement. The sound power comparisons are shown in Figures 11 and 12.

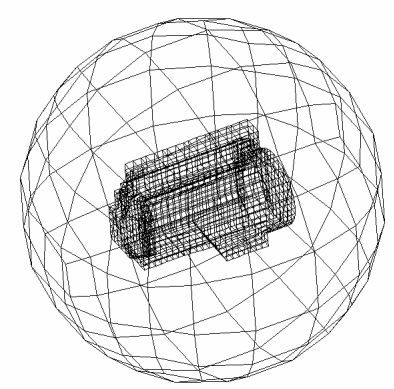

Figure 6 Sphere with 152 field points.

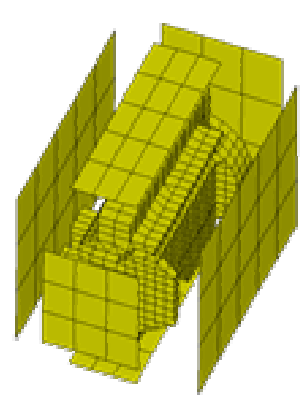

Figure 7 Six planes with 128 FP.

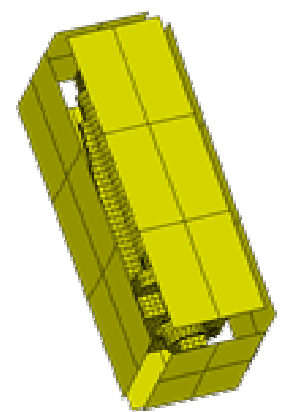

Figure 8 Six planes with 66 FP.

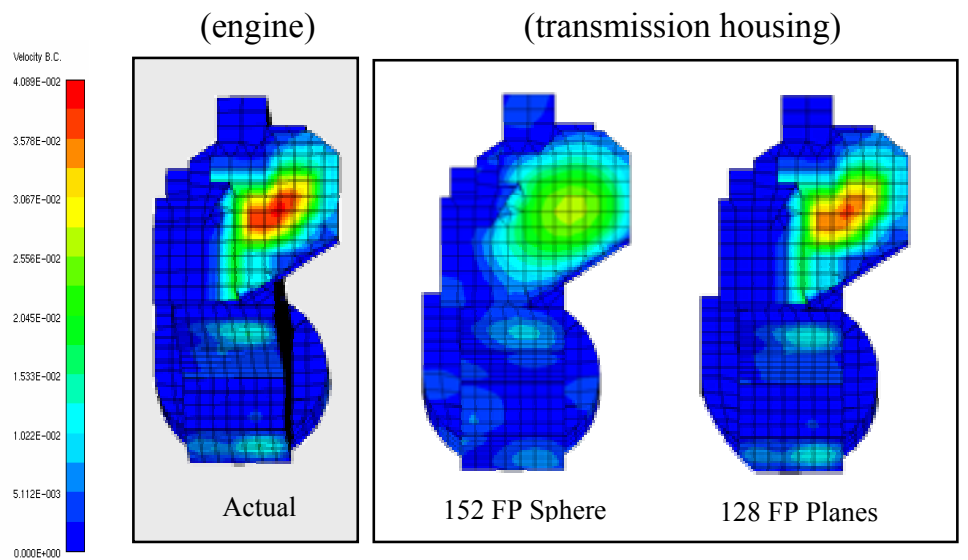

Figure 9 Comparison of reconstructed surface velocities at $220 \mathrm{~Hz}$. 

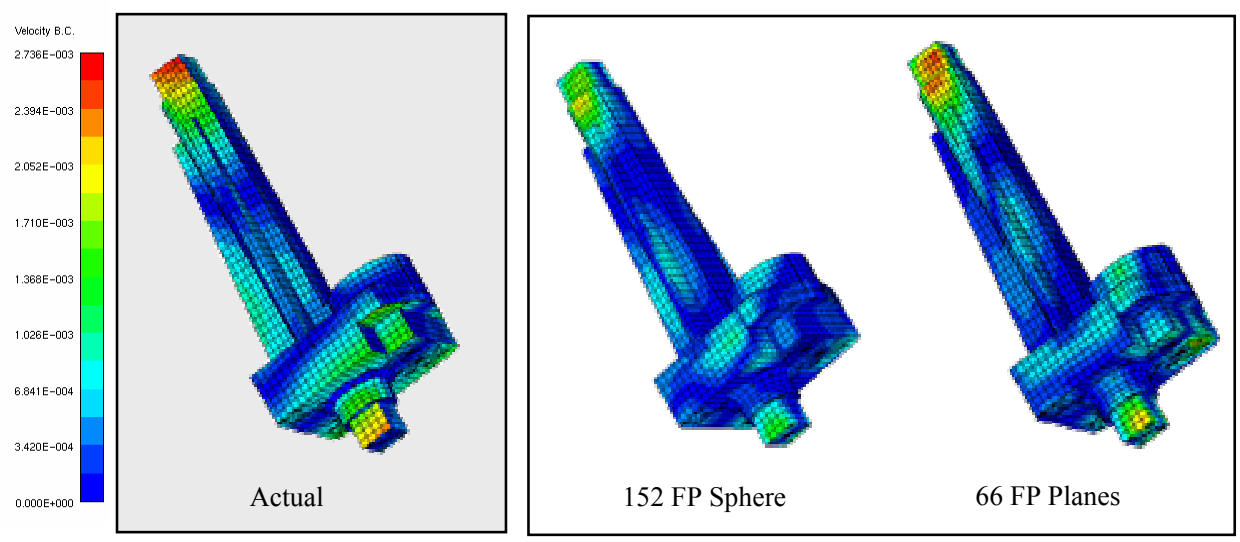

Figure 10 Comparison of reconstructed surface velocities at $1600 \mathrm{~Hz}$.

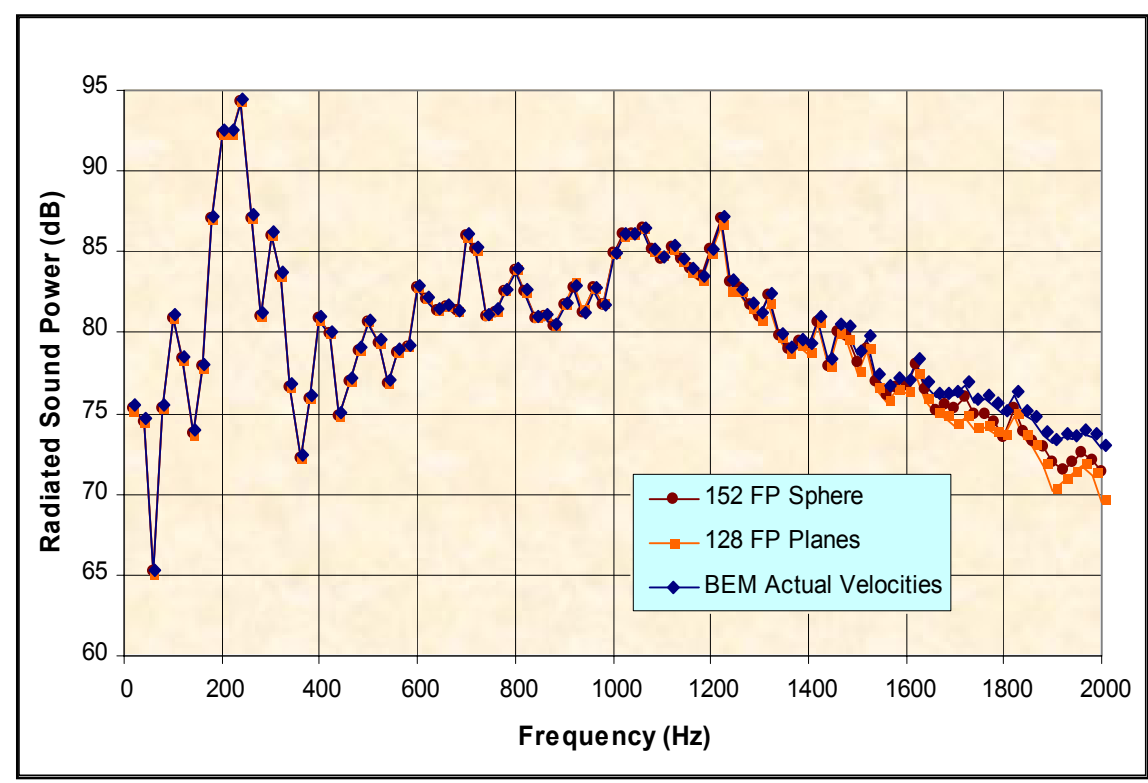

Figure 11 Comparison of reconstructed radiated sound power for the engine test case. 


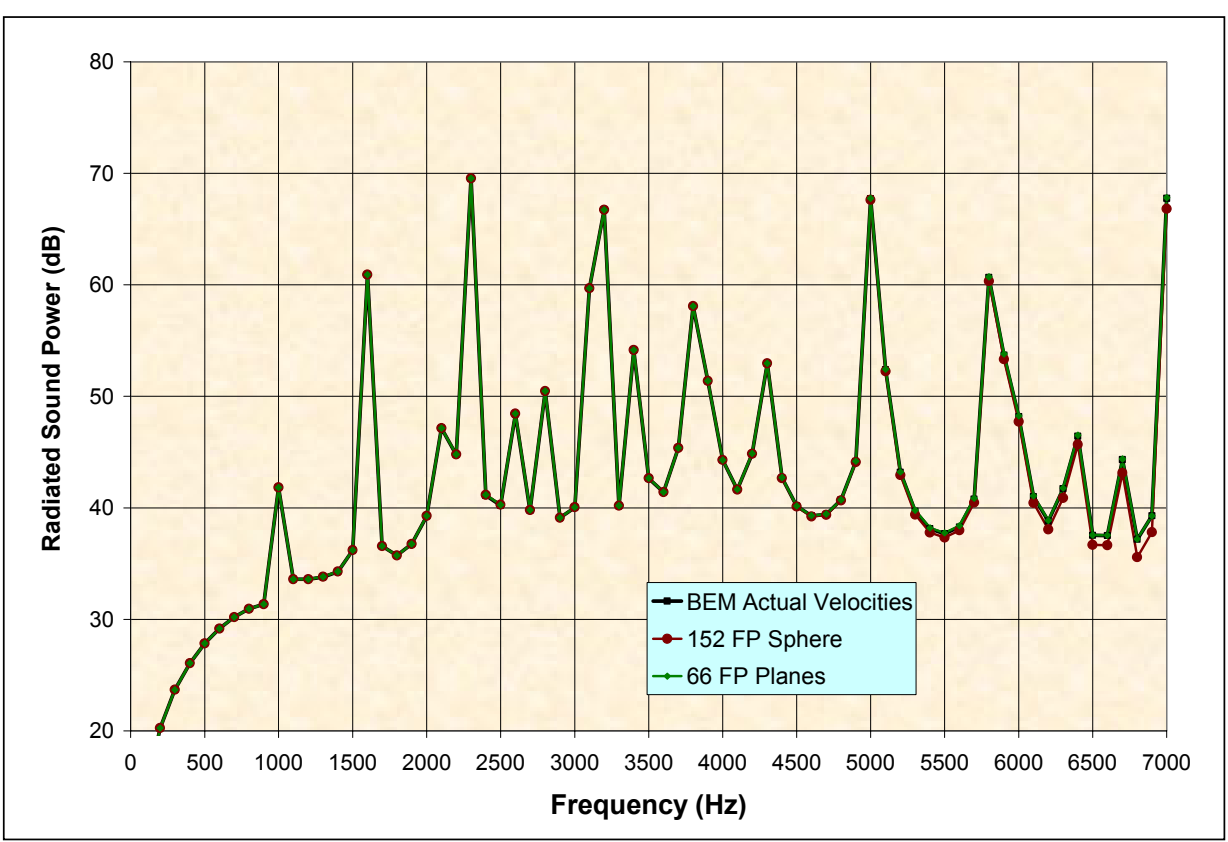

Figure 12 Comparison of radiated sound power for the transmission housing test case.

It can be observed from Figure 9 that using fewer field points arranged in six planes enclosing the engine produces a more accurate reconstruction than the 152 field point sphere. This is consistent with the previous discussion about field point selection. More field points do not necessarily make the reconstruction better. Figure 10 shows a similar result for the transmission housing in which a 66 field points arranged in six planes produced a similar reconstruction to that using a 152 field point sphere. It is interesting to observe the rank of matrix $A$ for each of the cases:

\begin{tabular}{|l|c|c|}
\hline & 152 FP Sphere & $\begin{array}{c}\text { FP Planes } \\
\text { (engine: 128 } \\
\text { housing: 66) }\end{array}$ \\
\hline $\begin{array}{l}\text { Transmission } \\
\text { housing }\end{array}$ & 63 & 64 \\
\hline Engine & 46 & 112 \\
\hline
\end{tabular}

Table 1 Rank of matrix $A$. 
From Table 1 it can be seen that the 152 field point sphere is not very effective since many points are being discarded in the reconstruction process. In the case of the transmission housing, using a more effective field point planes reduces the number of field points from 152 to 66 but the rank stays approximately the same. In the case of the engine, the number of field points is reduced from 152 to 128 but the rank increases from 46 to 112 . This shows that achieving a high rank of matrix $A$ can be done by arranging the field points correctly and not just by increasing the number of field points.

Although the accuracy of the reconstructed surface velocities varies depending which field point set is being used, the reconstructed sound power as shown in Figures 11 and 12 does not seem to be as sensitive. Actually it can be shown that a relatively inaccurate reconstructed surface velocity can still produce acceptable reconstructed radiated sound power.

Effects of SVD Tolerance. It has been mentioned that the SVD tolerance can be used to regularize the solution. Lowering the SVD tolerance will result in a higher $\operatorname{rank}(A)$ but will make matrix $A$ more ill-conditioned. Figure 13 shows the reconstructed surface vibration for the engine test case with SVD tolerances of 0.01 and 0.001 . In both cases, six planes and 128 field points were used.
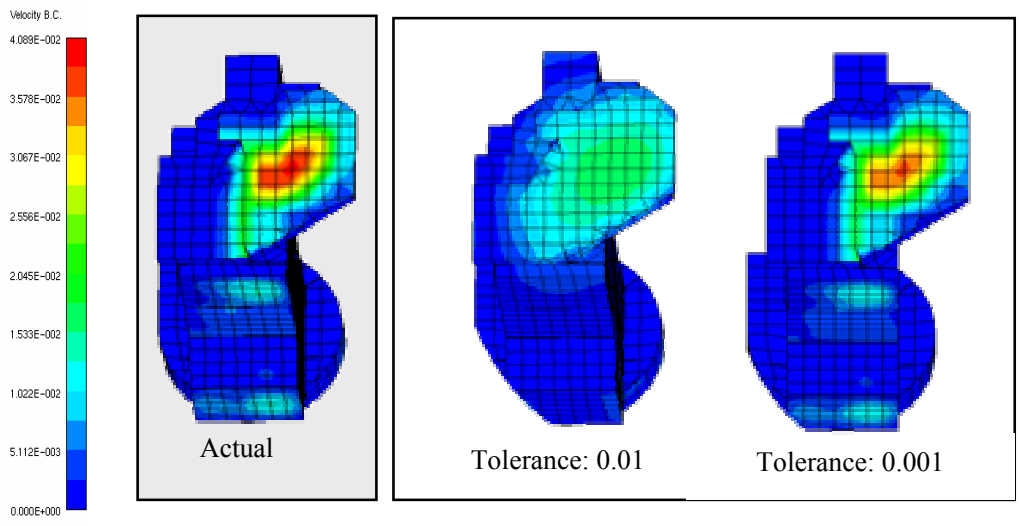

Figure 13 Reconstructed surface vibrations at $220 \mathrm{~Hz}$ for different SVD tolerances.

In this example, lowering the SVD tolerance from 0.01 to 0.001 changes the rank of matrix $A$ from 73 to 112 . However, it has to be noted that $A$ becomes more ill-conditioned since $\kappa_{2}(A)$ increases, meaning that the problem becomes more sensitive to errors. This would especially be the case if experimental errors were considered, as discussed below. 
The Use of Measured Surface Vibration Data. To demonstrate the use of measured surface vibration data, an actual velocity on one of the surface nodes was used as an input together with the field point sound pressure data; reconstructed surface velocities are shown in Figure 14. In this case an SVD tolerance of 0.01 was used.

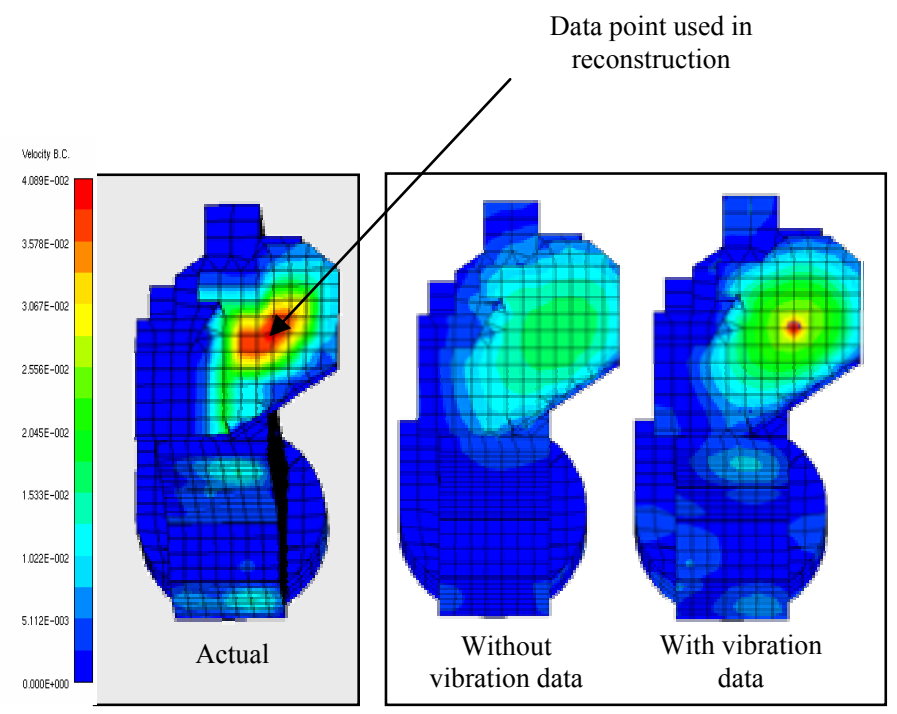

Figure 14 Reconstructed surface vibration at $220 \mathrm{~Hz}$ without and with the addition of measured vibration data.

From Figure 14 it is apparent that on the node which the velocity was specified, the result is exact. What is interesting is that using only one surface vibration value also improves the resolution of secondary areas of vibration on the bottom part of the engine.

\subsection{With 'Artificial' Experimental Error}

To simulate experimental errors in the measurement of field point sound pressures, random $1.5 \mathrm{~dB}$ magnitude and $3^{\circ}$ phase errors were introduced into the sound pressure input data. A comparison of the reconstructed surface velocities is shown in Figure 15. It can be seen that the reconstruction process is highly sensitive to measurement errors. 

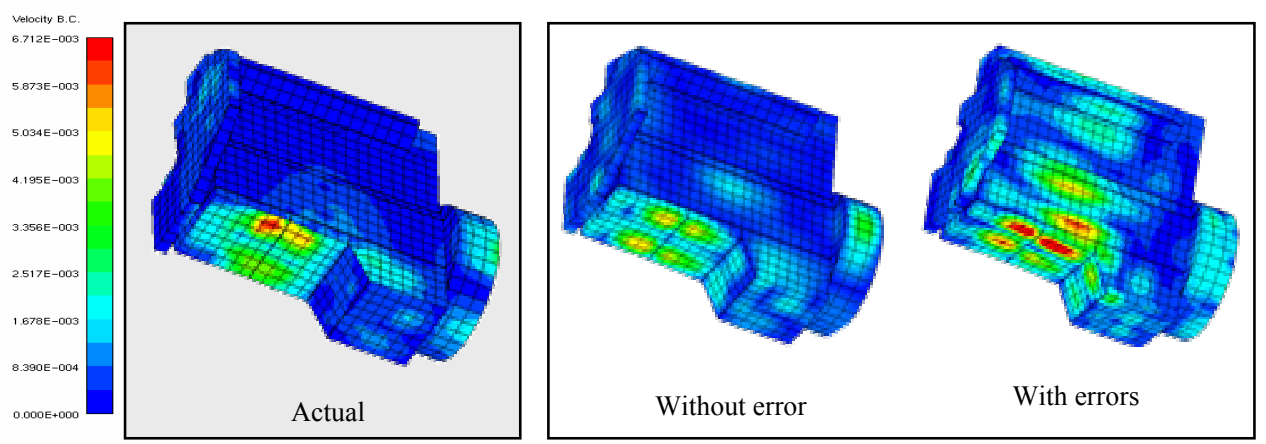

Figure 15 Reconstructed surface vibration at $440 \mathrm{~Hz}$ with the existence of 'artificial' experimental errors.

\subsection{With Real Experimental Error}

To include the actual effect of experimental errors, a surface vibration reconstruction test case was performed on an engine cover with 2148 nodes. The sound pressure data was measured at 150 field points located approximately $7.3 \mathrm{~cm}$ above the engine cover. The experimental setup is shown in Figure 16.
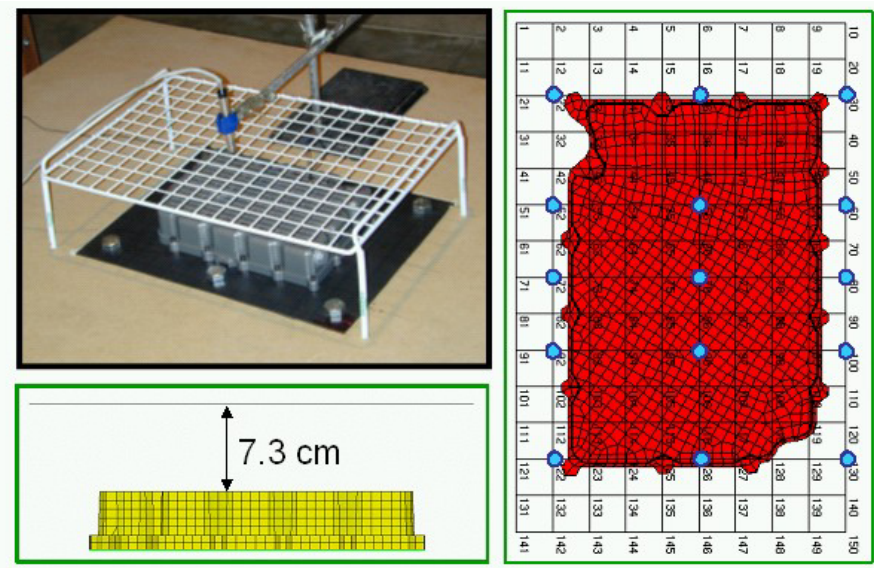

Figure 16 Setup for the engine cover test case with 150 field points grid.

To demonstrate the effect of experimental error described in Eqs. (8) and (12), the reconstructed surface vibration obtained using 15 and 150 field points is compared with the actual vibration in Figure 17. It can be seen that adding more field points can also accumulate the measurement errors which in turn will be amplified by $\kappa_{2}(A)$. 


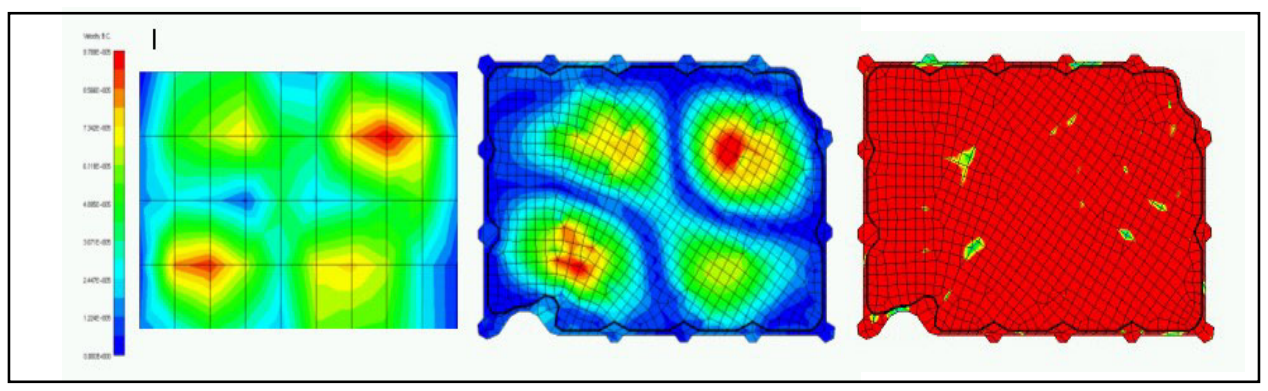

Figure 17 Surface vibration at $3000 \mathrm{~Hz}$ : actual (left), reconstructed using 15 field points (middle), reconstructed using 150 field points (right).

\section{Summary}

Reconstruction of the surface velocity of a sound source is valuable because it pinpoints the modes that are dominating the surface vibration. Once the surface velocity has been reconstructed, it can then be used to predict the sound pressure field and the radiated sound power which may be more valuable than the surface velocity reconstruction itself.

Since the solution to the inverse problem is generally not unique, a regularization process must be used to obtain the best solution. Two approaches have been discussed: a truncated SVD and Tickonov regularization. Using a truncated SVD produces a better conditioned matrix $A$. However the rank may be reduced. Hence, selecting a tolerance parameter is a trade-off between regularization and the loss of information.

It has been shown [2] that the number of field points required for an accurate reconstruction is proportional to the frequency of the reconstruction. However, adding field points does not always insure better accuracy since the field points may not be linearly independent. As presented in this paper, a field point set should be both unique and complete.

Since the non-uniqueness problem can be detected by observing small singular values corresponding to specific field points, an algorithm can be developed to automatically discard those points and replace them with points that result in larger singular values. New field points can be added to the set until the rank of the acoustic transfer matrix is sufficiently high, which physically means that complete information about the sound source has been captured.

Although experimental errors cannot be avoided in real situations, it is important to know that the existence of errors will be amplified by the condition 
number of $A$. Therefore, it is especially important to select the field points so that the acoustic transfer matrix is better conditioned and has a higher rank.

Partial reconstruction is suggested for the case where only the vibration or the sound power from part of the source is of particular interest. Another way to improve the reconstruction accuracy is by using few measured surface vibration data. This additional information would be useful to regularize the solution and also to reduce the rank deficiency of the acoustic transfer matrix.

\section{Acknowledgments}

The authors would like to acknowledge the members of the Vibro-Acoustics Consortium and the Center for Computational Sciences of the University of Kentucky for their support of this work.

\section{References}

1. Wu, T. W., Boundary Element Acoustics, WIT Press, Southampton, Boston (2000).

2. Seybert, A. F., and Martinus, F., "Forward and Inverse Numerical Acoustics for NVH Applications", $9^{\text {th }}$ International Congress on Sound and Vibration, P714-1, Orlando, Florida, July 8-11 (2002).

3. Gardner, B. K., and Bernhard, R. J., "A Noise Source Identification Technique Using an Inverse Helmholtz Integral Equation Method", J. Vib., Acoust., Stress Reliability and Design, 110, 84-90 (1988).

4. Veronesi, W. A., and Maynard, J. D., "Digital Holographic Reconstruction of Sources with Arbitrarily Shaped Surfaces", J. Acoust. Soc. Am., 85, 588-598 (1989).

5. Bai, M. R., "Application of BEM-based Acoustic Holography to Radiation Analysis of Sound Sources with Arbitrarily Shaped Geoometries", J. Acoust. Soc. Am., 92, 533-549 (1992).

6. Kim, B. K., and Ih, J. G., "On the Reconstruction of the Vibro-Acoustic Field Over the Surface Enclosing an Interior Space using the Boundary Element Method", J. Acoust. Soc. Am., 100, 3003-3015 (1996).

7. Ih, J. G., and Kim, B. K., "On the Use of the BEM-based NAH for the Vibro-Acoustic Source Imaging on the Non-Regular Exterior Surfaces", Proceedings of Noise-Con 98, Ypsilanti, Michigan, 665-770 (1998).

8. Zang, Z. et al., "A Computational Acoustic Field Reconstruction Process Based on an Indirect Boundary Element Formulation", J. Acoust. Soc. Am, 108, 2167-2178 (2000).

9. Tournour, M., Cremers, L., and Guisset, P., "Inverse Numerical Acoustics Based on Acoustic Transfer Vectors", $7^{\text {th }}$ International Congress on Sound and Vibration, Garmisch - Partenkirchen, Germany, 2069-1076 (2000). 
10. Demmel, J. W., Applied Numerical Linear Algebra, University of California, SIAM Publishing Co., 1997.

11. Maynard, J. D., Williams, E. G., and Lee, Y., "Nearfield Acoustic Holography: I. Theory of Generalized Holography and the Development of NAH', J. Acoust. Soc. Am., 78(4), 1395-1413 (1985).

12. Maynard, J. D. and Veronesi, W. A., "Nearfield Acoustic Holography II. Holographic Reconstruction Algorithms and Computer Implementation", J. Acoust. Soc. Am., 81(5), 1307-1322 (1987).

13. Wiliams, E. G. and Dardy, H. D., "Generalized Nearfield Acoustical Holography for Cylindrical Geometry: Theory and Experiment", J. Acoust. Soc. Am., 81,389-407 (1987).

14. Bai, M. R., "Application of BEM-based Acoustic Holography to Radiation Analysis of Sound Sources with Arbitrarily Shaped Geometries", J. Acoust. Soc. of Am., 92,533-549 (1992).

15. Wang, Z., "Helmholtz Equation-Least Squares (HELS) Method for Inverse Acoustic Radiation Problems", Ph.D. Dissertation, Wayne State University, Detroit (1995).

16. Wang, Z., Wu, S. F., "Helmholtz Equation-Least-Squares Method for Reconstructing the Acoustic Pressure Field", J. Acoust, Soc. Am., 102(4), 2020-2032 (1997).

17. Wu, S. F., "On Reconstruction of Acoustic Pressure Fields Using the Helmholtz Equation Least Squares Method", J. Acoust. Soc. Am, 107(5), 2511-2522 (2000).

18. Tickonov, A. N., "Solution of Incorrectly Formulated Problems and the Regularization Method", Soviet Math. Dokl, 4, pp. 1035-1038 (1963).

19. Hansen, P. C., and O'Leary, P. O, "The Use of the L-Curve in the Regularization of Discrete Ill-Posed Problems", SIAM J. Sci. Comput., 14, 1487-1503 (1993).

\section{Contact}

For additional information concerning this article and related questions, please contact Andy Seybert at 859-257-6336 x 80645 or via email to seybert@engr.uky.edu. 